Proceedings of the 9th Workshop on Quantum Chaos and Localisation Phenomena, May 24-26, 2019, Warsaw, Poland

\title{
Fock Space Localization of Many-Body States in the Tilted Bose-Hubbard Model
}

\author{
M. Schneider ${ }^{a}$, A. Rodríguez ${ }^{b}$ And A. Buchleitner ${ }^{a, *}$ \\ ${ }^{a}$ Physikalisches Institut, Albert-Ludwigs-Universität Freiburg, \\ Hermann-Herder-Str. 3, D-79104, Freiburg, Germany \\ ${ }^{b}$ Departamento de Fisica Fundamental, Universidad de Salamanca, E-37008, Salamanca, Spain
}

\begin{abstract}
We analyze the eigenstates' Fock space structure in the tilted Bose-Hubbard Hamiltonian. Fock space localization is quantified in terms of finite-size generalized fractal dimensions. This approach allows us to monitor the parametric evolution of localization across avoided crossings in the energy spectrum. We identify a manifold of strongly localized eigenstates, both in Fock and configuration space, which persists in regimes where all system parameters (hopping, interaction, and tilt strengths) are comparable. For a fixed number of bosons, the localization properties of that manifold are independent of the lattice size. The influence of avoided crossings on the structure of the localized manifold is discussed in detail.
\end{abstract}

DOI: 10.12693/APhysPolA.136.834

PACS/topics: localisation in Fock space, level repulsion, fractal dimensions

\section{Introduction}

One of the challenging problems in modern quantum mechanics is the understanding and control of the dynamics of many-particle systems. When increasing the number of particles, the system's complexity increases, not only due to the exponential growth of the underlying Hilbert space, but most significantly because of the intricate interplay between many-body interactions and many-particle interference (see, e.g., Refs. [1-4] and references therein). Such complexity may manifest itself, e.g., in the emergence of spectral chaos [5-9] or in Hilbert space structure of eigenstates, which can exhibit unusual statistical properties [10-15].

Given the general convoluted nature of the time evolution of many-body systems, it is always of interest to investigate the possibility of engineering quantum states with potentially controllable dynamical properties $[16,17]$. The desired dynamical stability may follow from the robustness of the state structure against perturbations in the Hamiltonian parameters. Remarkably, such an example was found recently in the tilted Bose-Hubbard model [18-20], for which a set of states that are strongly localized in Fock and real space, and well embedded in the energy spectrum, exhibited a significant stability to dynamical changes in the tilt strength.

Motivated by the latter results, we study in detail the eigenstate structure of the tilted Bose-Hubbard Hamiltonian and its correlation with the energy spectrum. In order to analyze localization in Fock space, we make use of the quantifiers employed in multifractal analysis, namely the finite-size generalized fractal

\footnotetext{
* corresponding author; e-mail:

a.buchleitner@physik.uni-freiburg.de
}

dimensions, which have proven to be very useful to describe the complexity of many-body states in Hilbert space, in particular for interacting bosons [14, 15, 21]. In Sect. 2, we describe the model and formalism employed. The relation between energy level repulsion and localization in the space is briefly presented in Sect. 3. In Sect. 4, we identify a manifold of eigenstates with special localization properties and investigate its dependence upon the system parameters. Finally, we discuss our results in the context of earlier studies.

\section{Model and formalism}

The Bose-Hubbard Hamiltonian [22, 23] is an archetypical many-particle model that can be experimentally implemented with ultracold atoms in optical lattices. Nowadays, the optical lattices provide a powerful playground to explore the physics of interacting many-body quantum systems (see, e.g., Refs. [24-30] and references therein). In particular, the so-called tilted Bose-Hubbard model is a paradigmatic system for investigating theoretically and experimentally the phenomenology of Bloch oscillations (see, e.g., Refs. [31-35]).

The tilted Bose-Hubbard model describes interacting bosons hopping in a lattice with a constant energy difference between neighbouring sites, as depicted in Fig. 1. The corresponding Hamiltonian can be written as

$$
H=-J \sum_{\langle l, j\rangle} \hat{b}_{l}^{\dagger} \hat{b}_{j}+\frac{U}{2} \sum_{l} \hat{n}_{l}\left(\hat{n}_{l}-1\right)+F \sum_{l} \tilde{l} \hat{n}_{l},
$$

in terms of the on-site bosonic creation $\left(\hat{b}_{l}^{\dagger}\right)$, annihilation $\left(\hat{b}_{l}\right)$, and number $\left(\hat{n}_{l}\right)$ operators. The one-dimensional lattice spans $L$ sites and contains $N$ bosons in the presence of hard wall boundary conditions (HWBC). Only nearest neighbour hopping is allowed, and we consider the case a of symmetric tilt around the centre of 


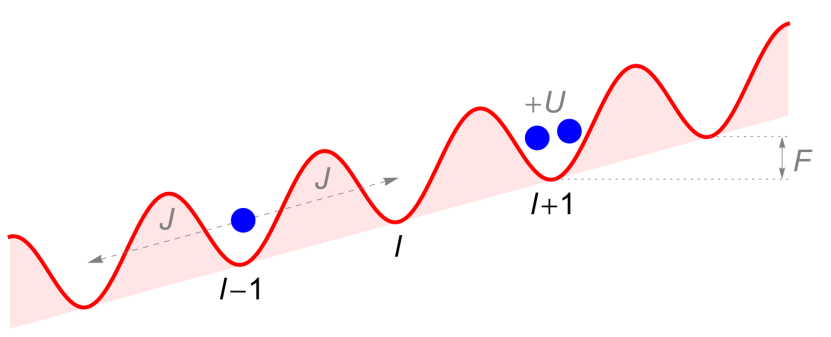

Fig. 1. Sketch of the tilted Bose-Hubbard model with nearest-neighbour hopping energy $J$, on-site interaction $U$ and tilt strength $F$.

the lattice, hence $\tilde{l} \equiv l-(L+1) / 2$. In the following, we set the interaction strength $U$ as the basic energy scale of the system.

Our basis of choice in the Hilbert space, of size $\mathcal{N}=\left(\begin{array}{c}N+L-1 \\ N\end{array}\right)$, will be given by the Fock states of the on-site density operators, $|\boldsymbol{n}\rangle \equiv\left|n_{1}, n_{2}, \ldots, n_{L}\right\rangle$, since they provide a direct description of the bosonic density in real space.

In the Fock basis, the interaction and tilt terms of Hamiltonian (1) are diagonal. Thus the eigenenergies of the system depend linearly on $F / U$ in the limit $J / U=0$. In this case, certain Fock states can be degenerate for all tilt strengths ${ }^{\dagger}$. Additionally, notable degeneracies between Fock states can occur at rational values of the ratio $F / U^{\ddagger}$, as shown in Fig. 2 .

On the other hand, the hopping term in the Hamiltonian couples different Fock states and is responsible for the off-diagonal elements in the matrix representation of $H$. For non-vanishing $J / U$, and finite $F / U>0$, all degeneracies in the spectrum are lifted, and the parametric evolution of the energy spectrum versus the tilt strength becomes populated by avoided crossings (cf. Fig. 2).

For arbitrary values of $J / U$ and $F / U$, the eigenstates of the tilted Bose-Hubbard Hamiltonian (TBHH) will have, in general, a non-trivial expansion in the Fock basis. In order to characterize their structure, and in particular their localization properties in Fock space, we make use of the tools of multifractal formalism [14, 15, 36], given a normalized eigenstate in the Fock basis,

$$
|\Psi\rangle=\sum_{\boldsymbol{n}} \psi_{\boldsymbol{n}}|\boldsymbol{n}\rangle
$$

\footnotetext{
${ }^{\dagger}$ For example, all Fock states that exhibit mirror symmetry about the middle of the lattice and that are related by a permutation of the on-site densities will have a common eigenenergy in the absence of hopping.

$\ddagger$ If we consider the simplest case of all Fock states that differ only in two on-site densities placed $l$ sites apart, one can see that degeneracies occur when $(F / U) l=1,2, \ldots, N-1$, where we have assumed $F / U>0$.
}

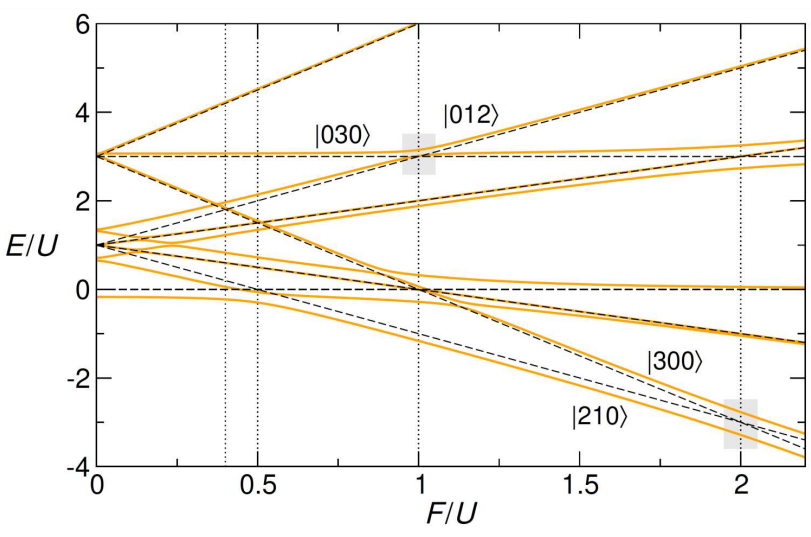

Fig. 2. Eigenenergies $E / U$ versus $F / U$ of the tilted Bose-Hubbard Hamiltonian with $L=N=3$ for $J / U=0$ (dashed black lines) and $J / U=0.15$ (solid orange lines). Vertical dotted lines mark the position of real crossings for $J / U=0$. For $J / U \neq 0$, a first order avoided crossing at $F / U=2$ and a second order avoided crossing at $F / U=1$ are highlighted by shaded areas, and the corresponding dominant Fock states involved in the crossings are indicated.

We define its $q$-moments, also known as generalized inverse participation ratios, as

$$
R_{q} \equiv \sum_{\boldsymbol{n}}\left|\psi_{\boldsymbol{n}}\right|^{2 q}, \quad q \in \mathbb{R}
$$

where different values of the exponent $q$ probe the properties of different ranges of the state's intensities $\left|\psi_{\boldsymbol{n}}\right|^{2}$. The scaling of such moments with the dimensionality of Hilbert space unveils the potentially extended, localized, or multifractal character of the state $|\Psi\rangle$. Nevertheless, for finite $\mathcal{N}$ a useful quantification of the degree of localization is provided by the quantities (so called finite-size generalized fractal dimensions):

$$
\widetilde{D}_{q} \equiv \frac{1}{1-q} \frac{\ln R_{q}}{\ln \mathcal{N}}
$$

Among the $\widetilde{D}_{q}$, we may single out the cases $q=1,2$ and $\infty$. The dimension $\widetilde{D}_{1}$ is related to the Shannon information entropy of the distribution of intensities $\left|\psi_{\boldsymbol{n}}\right|^{2}$. The value of $\widetilde{D}_{2}$ follows from the standard inverse participation ratio of the state, and for $q=\infty$ only the maximum value of the intensities is relevant, $\widetilde{D}_{\infty}=-\log _{\mathcal{N}} \max _{\boldsymbol{n}}\left|\psi_{\boldsymbol{n}}\right|^{2}$. The dimensions $\widetilde{D}_{q}$ are always monotonically decreasing functions of $q$ [37].

If a state covers Fock space homogeneously (i.e., $\left|\psi_{\boldsymbol{n}}\right|^{2}=\mathcal{N}^{-1}$ ), the latter dimensions have the trivial values $\widetilde{D}_{q}=1$, for all $q$. In contradistinction, a state localized on a single Fock state $\left|\boldsymbol{n}^{\prime}\right\rangle$ (i.e., $\psi_{\boldsymbol{n}}=\delta_{\boldsymbol{n}, \boldsymbol{n}^{\prime}}$ ) leads to $\widetilde{D}_{q}=0$ for $q>0$. A generic eigenstate of $H$ will exhibit values of $\widetilde{D}_{q}$ between 0 and 1 for $q>0$, as shown in Fig. 3. There, one can see how the degree of localization in Fock space is qualitatively reflected in the values of $\widetilde{D}_{q}$. 


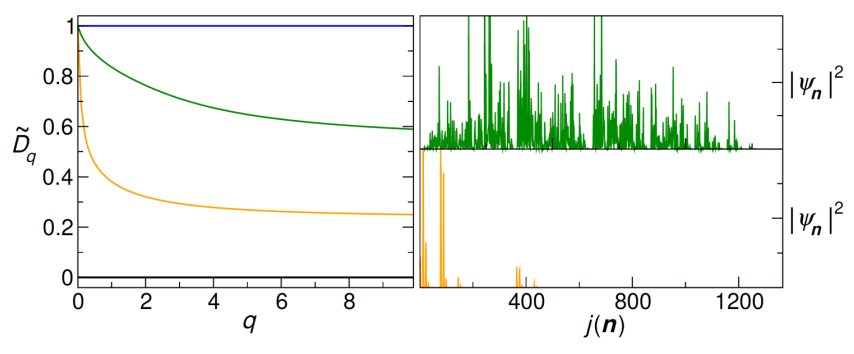

Fig. 3. (Left) Dimensions $\widetilde{D}_{q}$ versus $q$ in a finite Hilbert space of size $\mathcal{N}=1365$. The four lines shown correspond to a homogeneously extended state (blue), a fully localized state (black) and two eigenstates of the TBHH, the ground state (orange) and the 450th excited state (green). The parameters of the Hamiltonian were chosen to be $L=12, N=4, J / U=0.6, F / U=0.5$. (Right) Illustration of the intensities of the corresponding eigenstates in the Fock basis.

\section{Eigenstate structure around avoided crossings}

The level dynamics of the TBHH is strongly determined by avoided crossings, giving rise to spectral chaos in the system $[6,7,38]$. Such avoided crossings play a crucial role in the mixing of the Fock states, and hence in the Hilbert space structure of the eigenstates.

The emergence of avoided crossings can be described via a two-level approximation, in which two eigenstates $\left|\Phi_{1,2}\right\rangle$ of the Hamiltonian $H_{0}(F / U)$ (including in our case the interaction and tilt terms), whose energies $\varepsilon_{1,2}$ are degenerate for a certain $(F / U)_{0}$, become coupled by the hopping term $V(J / U)$ (first term in Eq. (1)), which induces a level repulsion around $(F / U)_{0}$ and the characteristic hyperbolic trajectories of the new eigenenergies as functions of $F / U$. The minimum energy gap at the avoided crossing is given by $\Delta E=2\left|\left\langle\Phi_{1}|V| \Phi_{2}\right\rangle\right|$. For the simplest avoid crossing, between $\left|\Phi_{1}\right\rangle=|\ldots, 0, N, 0, \ldots\rangle$ and $\left|\Phi_{2}\right\rangle=|\ldots, 0, N-1,1,0, \ldots\rangle$, one can straightforwardly obtain $\Delta E=2 J \sqrt{N}$, and the parametric width of the crossing region can be estimated to be $\Delta F \simeq 4 J \sqrt{N}[39]$.

In the two-level approach, the mixing of $\left|\Phi_{1,2}\right\rangle$ through the avoided crossing can be cast as a rotation,

$$
\begin{aligned}
& \left|\Psi_{1}\right\rangle=\cos (\theta / 2)\left|\Phi_{1}\right\rangle-\sin (\theta / 2)\left|\Phi_{2}\right\rangle, \\
& \left|\Psi_{2}\right\rangle=\sin (\theta / 2)\left|\Phi_{1}\right\rangle+\cos (\theta / 2)\left|\Phi_{2}\right\rangle,
\end{aligned}
$$

for $0<\theta<\pi$, and where $\tan \theta=2\left|\left\langle\Phi_{1}|V| \Phi_{2}\right\rangle\right| /\left(\varepsilon_{1}-\varepsilon_{2}\right)$. In our case, the angle $\theta$ simply parametrizes the change of $F / U$ through the crossing. Such a two-state treatment is only justified for small $J / U$ and cannot account for the level repulsion due to the mixing of Fock states which differ in more than one particle hopping (which we refer to as higher order avoided crossings (cf. Fig. 2)). In order to get a qualitative understanding of the effect imparted by the level repulsion on the parametric evolution of the dimensions $\widetilde{D}_{q}$, going beyond the twolevel approximation, we will assume that the underlying states involved in the crossing $\left|\Phi_{1,2}\right\rangle$ may already be superpositions of Fock states, and that their mixing is still roughly captured by Eqs. (5).

We consider two exemplary cases. First, let us assume

$$
\begin{aligned}
& \left|\Phi_{1}\right\rangle=|\boldsymbol{n}\rangle, \\
& \left|\Phi_{2}\right\rangle=\frac{1}{\sqrt{1+\delta^{2}}}\{|\boldsymbol{m}\rangle+\delta|\boldsymbol{o}\rangle\}, \quad \delta \in \mathbb{R},
\end{aligned}
$$

where $|\boldsymbol{n}\rangle,|\boldsymbol{m}\rangle$ and $|\boldsymbol{o}\rangle$ denote orthogonal Fock states. Making use of Eqs. (3)-(5), the dimensions $\widetilde{D}_{q}$ in the Fock basis for the eigenstates $\left|\Psi_{1,2}\right\rangle$ can be straightforwardly calculated, and the corresponding evolution through the avoided crossing is shown in the upper panels of Fig. 4. At the centre of the avoided crossing (i.e., $\theta=\pi / 2$ ), the eigenstates have the form

$$
\begin{aligned}
& \left|\Psi_{1}\right\rangle=\frac{1}{\sqrt{2}}\left[|\boldsymbol{n}\rangle+\frac{1}{\sqrt{1+\delta^{2}}}|\boldsymbol{m}\rangle+\frac{\delta}{\sqrt{1+\delta^{2}}}|\boldsymbol{o}\rangle\right] \\
& \left|\Psi_{2}\right\rangle=\frac{1}{\sqrt{2}}\left[|\boldsymbol{n}\rangle-\frac{1}{\sqrt{1+\delta^{2}}}|\boldsymbol{m}\rangle-\frac{\delta}{\sqrt{1+\delta^{2}}}|\boldsymbol{o}\rangle\right] .
\end{aligned}
$$

As a consequence of the mixing, the states acquire more non-zero Fock coefficients as compared to their asymptotic forms away from the crossing (Eqs. (6)); the states become more extended in Fock space and correspondingly the values of $\widetilde{D}_{q}$ are enhanced when traversing the crossing, as can be seen in Fig. 4.

If, instead, we consider the case

$$
\begin{aligned}
& \left|\Phi_{1}\right\rangle=\frac{1}{\sqrt{2+\delta^{2}}}\{|\boldsymbol{n}\rangle-|\boldsymbol{m}\rangle+\delta|\boldsymbol{o}\rangle\}, \quad \delta \in \mathbb{R}, \\
& \left|\Phi_{2}\right\rangle=\frac{1}{\sqrt{2}}\{|\boldsymbol{n}\rangle+|\boldsymbol{m}\rangle\},
\end{aligned}
$$

one can check that the mixing induced by the avoided crossing leads to an enhanced localization in Fock space, as can be observed in the lower panels of Fig. 4 .

This simple analysis can help us understand the parametric evolution of the dimensions $\widetilde{D}_{q}$ and correlate it with the occurrence of avoided crossings in the energy spectrum. Furthermore, the appearance of a characteristic form of the parameter dependence, such as observed in Fig. 4, can be linked to a particular underlying Fock structure of the states which undergo the avoided crossing. We also emphasize that all $\widetilde{D}_{q}$ for $q>0$ exhibit the same qualitative behaviour.

\section{Manifold of localized eigenstates}

In order to have a global picture of the influence of the tilt on the eigenstate structure, we show in Fig. 5 the parametric evolution (as a function of $F / U$ ) of $\widetilde{D}_{1}$ for all eigenstates, correlated with the level dynamics, for a system with $L=N=4(\mathcal{N}=35)$ and $J / U=0.05$. The eigenenergies are given in terms of the energy density

$$
\epsilon \equiv \frac{E-E_{\min }}{E_{\max }-E_{\min }},
$$

which provides a convenient representation of the spectrum for varying tilt strength. 


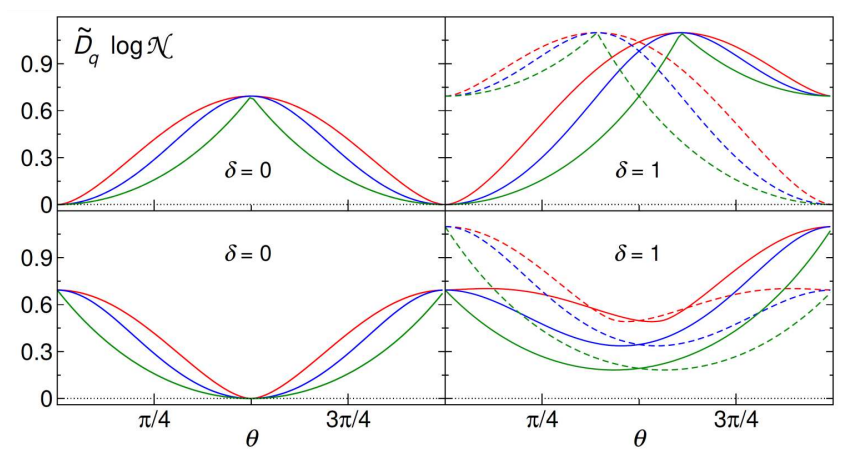

Fig. 4. Exemplary behaviour of the evolution of $\widetilde{D}_{q}$ $(q=1$ (red), $q=2$ (blue), $q=\infty$ (green)) for the states $\left|\Psi_{1}\right\rangle$ (solid lines) and $\left|\Psi_{2}\right\rangle$ (dashed lines) through an avoided crossing. The upper and lower panels correspond to the cases considered in Eqs. (6) and (8), respectively, for $\delta=0$ (left) and $\delta=1$ (right). Note that for $\delta=0$ the trajectories of both states coincide.

The evolution of $\widetilde{D}_{1}$ unveils the dependence of Fockspace localization on $F / U$, which, as can be observed, is strongly determined by the presence of avoided crossings. The qualitative behaviour shown in Fig. 4 and discussed in Sect. 3 can be clearly identified in Fig. 5 .

As can be expected for small hopping strength, some eigenstates will be strongly localized around single Fock states. In Fig. 5 we highlight, in particular, those eigenstates which have more than $90 \%$ of their norm concentrated on a single Fock state with all bosons on one site, and we label them as $\left|\Psi_{4000}\right\rangle,\left|\Psi_{0400}\right\rangle,\left|\Psi_{0040}\right\rangle$, and $\left|\Psi_{0004}\right\rangle$, indicating the dominant Fock state. This manifold of eigenstates exhibit the strongest localization (lowest $\widetilde{D}_{1}$ ) for $0<F / U<1$. In this range, states $\left|\Psi_{4000}\right\rangle$ and $\left|\Psi_{0400}\right\rangle$ (green and orange) go through several very narrow avoided crossings that do not change their localization properties. The first visible small change of these states' structure occurs at $F / U=1$, where a third order avoided crossing happens (i.e., three particle hopping: state $\left|\Psi_{4000}\right\rangle$ crosses with $\left|\Psi_{1300}\right\rangle$, etc.). At $F / U=3 / 2$ and $F / U=2$, two second order avoided crossings take place. The strongest distortion of localization for $\left|\Psi_{4000}\right\rangle,\left|\Psi_{0400}\right\rangle$ and $\left|\Psi_{0040}\right\rangle$ occurs at $F / U=3$, due to a first order avoided crossing. The state with the highest energy, $\left|\Psi_{0004}\right\rangle$ (red), does not undergo any avoided crossing and conserves its localization properties over the whole range of $F / U$.

Since the hopping strength controls Fock space mixing, localization and hence $\widetilde{D}_{q}$ should naturally be expected to depend on the value of $J / U$. (The larger the hopping strength, the stronger the level repulsion, the greater the relevance of higher order crossings, and thus the shorter the $F / U$-range within which localization of the eigenstates mentioned above persists [39].) The most interesting scenario to be considered is that in which all parameters in the Hamiltonian, $J, U$, and $F$, are comparable, and the Fock space is sufficiently large. The evolution of $\widetilde{D}_{q}$ for the whole spectrum, as in Fig. 5, gets

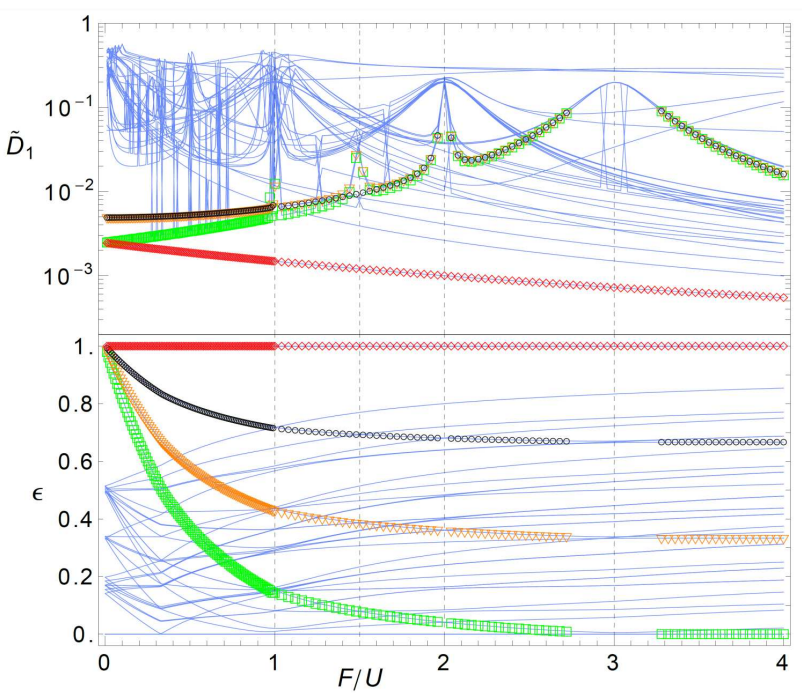

Fig. 5. $\widetilde{D}_{1}$ (upper panel, Eq. (4)) and energy density $\epsilon$ (lower panel, Eq. (9)) versus $F / U$ for the TBHH with $L=N=4$ and $J / U=0.05$. Colour symbols highlight those eigenstates that have more than $90 \%$ of their norm concentrated on a single Fock state, as follows: $\left|\Psi_{4000}\right\rangle$ (green), $\left|\Psi_{0400}\right\rangle$ (orange), $\left|\Psi_{0040}\right\rangle$ (black) and $\left|\Psi_{0004}\right\rangle$ (red). Vertical dashed lines mark the position of major avoided crossings. The higher density of points between 0 and 1 is due to a finer resolution in $F / U$.

however increasingly involved for large Hilbert spaces. In order to assess the existence of eigenstates with special localization properties, it is more effective to visualize a scatter plot $\widetilde{D}_{q}$ versus $\epsilon$ for different values of the tilt strength.

In Fig. 6, we show the evolution of the scatter plot $\widetilde{D}_{2}$ versus $\epsilon$ for $J / U \simeq 0.4$ and increasing values of the tilt, $F / U \in\{0.10,0.35,0.60\}$, for a system with $N=4$ and system sizes $L=8(\mathcal{N}=330)$ and $L=12(\mathcal{N}=1365)$. Those eigenstates with at least $75 \%$ of their norm localized on a Fock state with all bosons on one site are highlighted in red.

As can be observed, a manifold of $L$ strongly localized eigenstates is clearly identifiable for both system sizes. Each state of the manifold can still be associated with a Fock state with all bosons on one site. We refer to all states that are not part of such manifold as the bulk. The $\widetilde{D}_{2}$ values of the bulk and the localized manifold differ by an order of magnitude, even for values of $F / U$ comparable to $J / U$. Furthermore, it is apparent that all bulk states exhibit similar localization properties, which give rise to a well defined cluster of $\widetilde{D}_{2}$ values that gets more recognisable the larger $L$. As $F / U$ increases, the localized states spread out in energy and eventually (almost) fully overlap with the bulk along the $\epsilon$ axis. In the course of this spreading, the states of the manifold go through a large number of narrow avoided crossings that do not affect their eigenstate structure $\left(\widetilde{D}_{2}\right.$ does not change noticeably). 


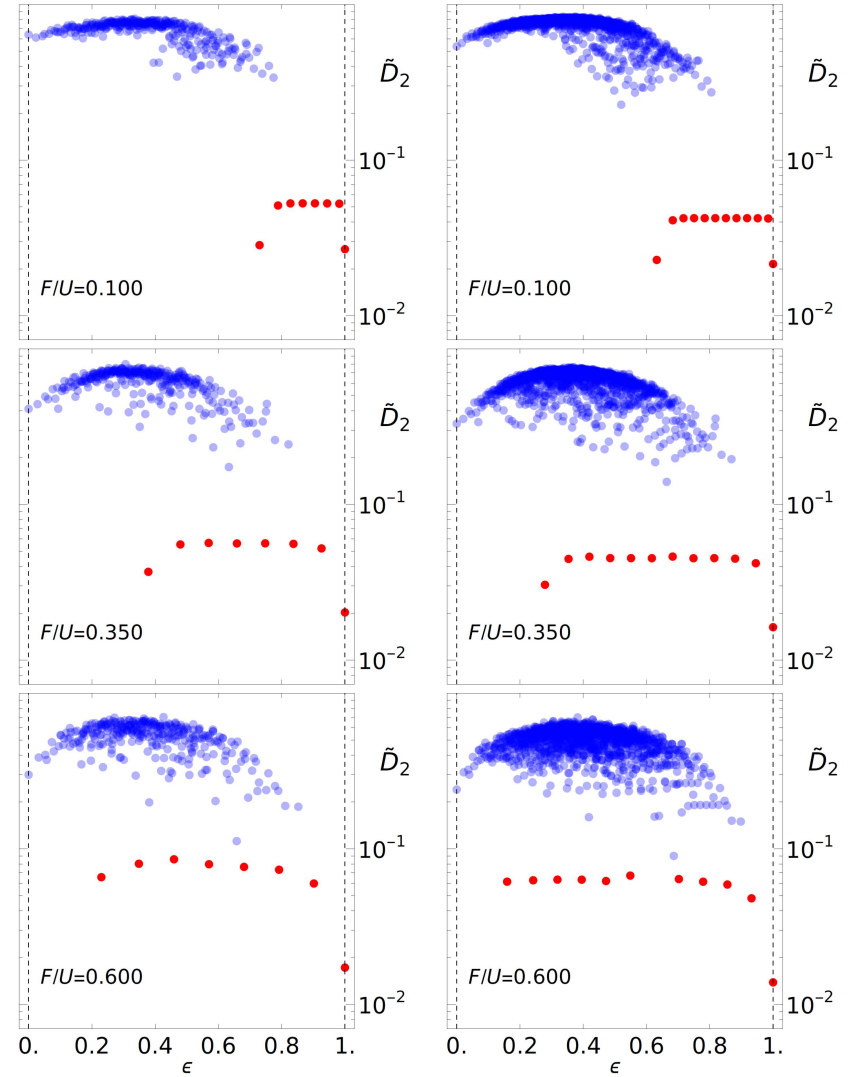

Fig. 6. Scatter plots $\widetilde{D}_{2}$ versus $\epsilon$ for $J / U=0.3981$ and three different values of $F / U$ (increasing from top to bottom) for the TBHH with $N=4$ and $L=8$ (left parts) and $L=12$ (right parts). The states marked in red have more than $75 \%$ of their norm localized on the subspace of Fock states with all bosons on one site. The inhomogeneity of localization within the red manifold is due to the use of HWBC, which reduce further the Fock space connectivity of the two Fock states with all bosons on one edge of the lattice. Note that in the bottom right panel, one state of the manifold is undergoing an avoided crossing and breaks the $75 \%$ localization condition.

In Fig. 7, we provide a different perspective of the transit of the localized manifold through the bulk of the spectrum as a function of the tilt strength. There, the level dynamics can be correlated with the evolution of $\widetilde{D}_{\infty}$. The orange, red, and blue symbols highlight the eigenstates with more than $90 \%$, between $90 \%$ and $80 \%$, and between $80 \%$ and $70 \%$, respectively, of their norm localized on the Fock states with all bosons on one site. The localized manifold induces characteristic bulk-traversing linear trajectories in the evolution of the energy spectrum as a function of $F / U$, as reported in Refs. [18-20]. Such linear dependence stems from the conserved centre of mass of the bosonic density in the localized eigenstates, as it follows from the application of Hellmann-Feynman's theorem [40] to the TBHH, $\partial E / \partial F=\left\langle\sum_{l} \tilde{l} \hat{n}_{l}\right\rangle$.

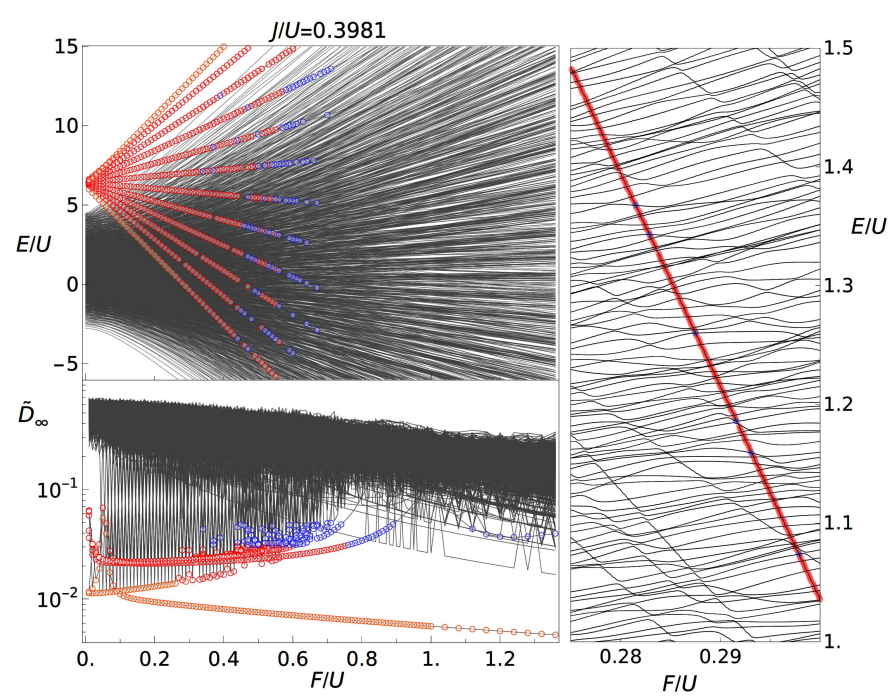

Fig. 7. Energy $E / U$ and $\widetilde{D}_{\infty}$ versus $F / U$ for the TBHH with $N=4, L=12$, and $J / U=0.3981$. The right panel shows a zoom of the level dynamics. The orange, red, and blue symbols highlight the eigenstates with more than $90 \%$, between $90 \%$ and $80 \%$, and between $80 \%$ and $70 \%$, respectively, of their norm localized on the Fock states with all bosons on one site.

As $F / U$ is increased, localization in the manifold is eventually lost. By following the evolution of $\widetilde{D}_{\infty}$ in Fig. 7, one may estimate that the localized manifold persists up to $F / U \approx 0.6-0.7$. For the larger value of $J / U \simeq 0.4$ there considered, higher order avoided crossings (note in particular the third order crossing at $F / U=1)$ play a significant role in the delocalization of the manifold (see also Ref. [39]), which can not be solely attributed to first order hopping processes, as suggested in Ref. [20].

\section{Final discussion}

We have studied the localization properties in Fock space of the many-body eigenstates of the tilted Bose-Hubbard model. In order to investigate the state structure, an approach based on generalized fractal dimensions $\widetilde{D}_{q}$ to quantify the degree of localization in finite Hilbert spaces was used. We have described the localization properties of states undergoing an avoided crossing, and identified these in the parametric evolution of $\widetilde{D}_{q}[39]$.

In agreement with the results of Refs. [18-20], we have shown that there exists a special manifold of states which exhibit robust localization properties well separated from those of the rest of the spectrum. Such states are strongly linked to the Fock states with all bosons on onesite. They exhibit also localization of the bosonic density in real space. The localized manifold survives in regimes, where $J, U$, and $F$ are comparable, i.e., in the presence 
of a non-trivial interplay between hopping, interaction and tilt. Moreover, for a fixed number of bosons, the dependence of localization on $F / U$ is independent of the number of lattice sites.

The Fock states with all particles on one site have minimal connectivity in Fock space. For nearest-neighbour hopping, they couple only to 2 (or even 1 , if they directly probe the HWBC) other Fock states. In a geometrical visualization of Fock space (i.e., if Hilbert space is displayed as a network whose nodes are Fock states which are linked to each other via the hopping term in Hamiltonian (1)), these states are always vertices located on the external boundary of a complex network. Hence they have the lowest possible coordination number. This fact is independent of the total number of bosons and lattice sites. Therefore, the existence of the localized manifold in the TBHH should be a generic feature for any values of $N$ and $L$.

In Ref. [20], it was suggested that one may also speak of a second set of states with special localization properties that are associated with Fock states of the form $|\ldots, 0, N-1,1,0, \ldots\rangle$. While we did not look specifically at the eigenstates emerging from that set of Fock states, the analysis of $\widetilde{D}_{q}$ (cf. Fig. 6) signals that their localization properties must be closer to those of the bulk of the spectrum, and are not so pronounced as for what we here called the localized manifold.

Regarding the range of $F / U$ within which the localized manifold exists, an estimation for the upper border, $F_{t} \simeq U(N-1)-2 J \sqrt{N}$, was given in Ref. [20], based on the analysis of the width of the first order avoided crossing between states of the form $\left|\Psi_{\ldots 0, N, 0 \ldots}\right\rangle$ and $\left|\Psi_{\ldots 0, N-1,1,0 \ldots}\right\rangle$, which worked qualitatively well for the parameters there chosen. For $N=4$ and $J / U \simeq 0.4$, the latter equation yields an overestimation for $F_{t}$ in view of Fig. 7 , where the localized manifold arguably persists until $F_{t} / U \simeq 0.6-0.7$. Nevertheless, a precise criterion to characterize the loss of localization would be necessary in order to unambiguously define a threshold value for the tilt strength. This requires a more detailed study that neither here nor in Ref. [20] was carried out.

The results here presented indicate that the role of higher order avoided crossings (cf. third order avoided crossing at $F / U=1$ in Fig. 7), perhaps via the use of higher order degenerate perturbation theory, will have to be included in such an analysis. Additionally, the investigation of systems with constant lattice size and variable $N$, as well as with constant filling factor $N / L$, would be desirable.

\section{Acknowledgments}

The authors acknowledge support by the state of Baden-Württemberg through bwHPC and by the German Research Foundation (DFG) through Grant No. 402552777.

\section{References}

[1] T. Brünner, G. Dufour, A. Rodríguez, A. Buchleitner, Phys. Rev. Lett. 120, 210401 (2018).

[2] T. Brünner, Ph.D. Thesis, AlbertLudwigs-Universität Freiburg, 2018.

[3] T. Giordani, F. Flamini, M. Pompili, N. Viggianiello, N. Spagnolo, A. Crespi, R. Osellame, N. Wiebe, M. Walschaers, A. Buchleitner, F. Sciarrino, Nat. Photon. 12, 173 (2018).

[4] C. Dittel, G. Dufour, M. Walschaers, G. Weihs, A. Buchleitner, R. Keil, Phys. Rev. Lett. 120, 240404 (2018).

[5] F. Haake, Quantum Signatures of Chaos, Springer, Berlin 2004.

[6] A.R. Kolovsky, A. Buchleitner, J. Mod. Opt. 51, 999 (2004).

[7] A.R. Kolovsky, A. Buchleitner, Europhys. Lett. 68, $632(2004)$.

[8] C. Kollath, G. Roux, G. Biroli, A.M. Läuchli, J. Stat. Mech. Theory Exp. 2010, P08011 (2010).

[9] M. Hiller, T. Kottos, T. Geisel, Phys. Rev. A 73, 061604 (2006).

[10] Y.Y. Atas, E. Bogomolny, Phys. Rev. E 86, 021104 (2012).

[11] Y.Y. Atas, E. Bogomolny, Philos. Trans. R. Soc. A 372, 20120520 (2014).

[12] D.J. Luitz, F. Alet, N. Laflorencie, Phys. Rev. Lett. 112, 057203 (2014).

[13] D.J. Luitz, N. Laflorencie, F. Alet, Phys. Rev. B 91, 081103 (2015).

[14] J. Lindinger, M.Sc. Thesis, Albert-Ludwigs-Universität Freiburg, 2017.

[15] J. Lindinger, A. Buchleitner, A. Rodríguez, Phys. Rev. Lett. 122, 106603 (2019).

[16] J. Zakrzewski, A. Buchleitner, D. Delande, Z. Phys. B 103, 115 (1997)

[17] A. Buchleitner, D. Delande, J. Zakrzewski, Phys. Rep. 368, 409 (2002).

[18] H. Venzl, T. Zech, B. Oleś, M. Hiller, F. Mintert, A. Buchleitner, Appl. Phys. B 98, 647 (2010).

[19] H. Venzl, Ph.D. Thesis, Albert-Ludwigs-Universität Freiburg, 2011.

[20] M. Hiller, H. Venzl, T. Zech, B. Oleś, F. Mintert, A. Buchleitner, J. Phys. B 45, 095301 (2012).

[21] E. Bogomolny, "Multifractality in simple systems" (presentation at the conference Complex patterns in wave functions: drums, graphs, and disorder, Newport Pagnell (UK) 2012).

[22] H.A. Gersch, G.C. Knollman, Phys. Rev. 129, 959 (1963).

[23] M.P.A. Fisher, P.B. Weichman, G. Grinstein, D.S. Fisher, Phys. Rev. B 40, 546 (1989).

[24] K.V. Krutitsky, Phys. Rep. 607, 1 (2016).

[25] I. Bloch, J. Dalibard, W. Zwerger, Rev. Mod. Phys. 80, 885 (2008).

[26] I. Bloch, J. Dalibard, S. Nascimbène, Nat. Phys. 8 , 267 (2012). 
[27] M. Lewenstein, A. Sanpera, V. Ahufinger, B. Damski, A. Sen, U. Sen, Adv. Phys. 56, 243 (2007).

[28] W.S. Bakr, A. Peng, M.E. Tai, R. Ma, J. Simon, J.I. Gillen, S. Folling, L. Pollet, M. Greiner, Science 329, 547 (2010).

[29] M. Greiner, O. Mandel, T. Esslinger, T.W. Hänsch, I. Bloch, Nature 415, 39 (2002).

[30] M.A. Cazalilla, R. Citro, T. Giamarchi, E. Orignac, M. Rigol, Rev. Mod. Phys. 83, 1405 (2011).

[31] A.R. Kolovsky, H.J. Korsch, Int. J. Mod. Phys. B 18, 1235 (2004).

[32] T. Hartmann, F. Keck, H.J. Korsch, S. Mossmann, New J. Phys. 6, 2 (2004).

[33] M. Gustavsson, E. Haller, M.J. Mark, J.G. Danzl, G. Rojas-Kopeinig, H.C. Nägerl, Phys. Rev. Lett. 100, 080404 (2008).
[34] F. Meinert, M.J. Mark, E. Kirilov, K. Lauber, P. Weinmann, M. Gröbner, H.C. Nägerl, Phys. Rev. Lett. 112, 193003 (2014).

[35] Z.A. Geiger, K.M. Fujiwara, K. Singh, R. Senaratne, S.V. Rajagopal, M. Lipatov, T. Shimasaki, R. Driben, V.V. Konotop, T. Meier, D.M. Weld, Phys. Rev. Lett. 120, 213201 (2018).

[36] A. Rodriguez, L.J. Vasquez, K. Slevin, R.A. Römer, Phys. Rev. B 84, 134209 (2011).

[37] H.G.E. Hentschel, I. Procaccia, Physica D Nonlin. Phenom. 8, 435 (1983).

[38] A. Buchleitner, A.R. Kolovsky, Phys. Rev. Lett. 91, 253002 (2003).

[39] M.T.V. Schneider, B.Sc. Thesis, Albert-LudwigsUniversität Freiburg, 2019.

[40] R.P. Feynman, Phys. Rev. 56, 340 (1939). 